\title{
Efficacy of Moxifloxacin-Based Triple Therapy as Second-Line Treatment for Helicobacter pylori Infection
}

\author{
Jae Hee Cheon, ${ }^{*}$ Nayoung Kim, ${ }^{* \dagger}$ Dong Ho Lee, ${ }^{\star \dagger}$ Jung Mogg Kim, ${ }^{\ddagger}$ Joo Sung Kim, ${ }^{*}$ Hyun Chae Jung ${ }^{*}$ and \\ In Sung Song*
}

"Department of Internal Medicine, Liver Research Institute, Seoul National University College of Medicine, Seoul; 'Department of Internal Medicine, Seoul National University Bundang Hospital, Seongnamsi, Gyeonggi-do; :Department of Microbiology and Institute of Biomedical Science, Hanyang University College of Medicine, Seoul, Korea

\author{
Keywords \\ Helicobacter pylori, moxifloxacin, second-line, \\ quadruple.
}

Reprint requests to: Nayoung Kim, Department of Internal Medicine, Seoul National University Bundang Hospital, 300 Gumi-dong, Bundang-gu, Seongnam, Kyunggi-do, 463-707, South Korea. E-mail: nayoungkim49@empal.com

\begin{abstract}
Background and Aim: Metronidazole and tetracycline-based second-line quadruple therapy, widely used for Helicobacter pylori infection, often ends up in failure due to antibiotic resistance and poor compliance in Korea. Our aim is to evaluate the efficacy and tolerability of moxifloxacin-based triple therapy as an alternative second-line treatment for $\mathrm{H}$. pylori infection.

Methods: The subjects consisted of 85 patients infected with H. pylori, in whom initial proton pump inhibitor triple therapy had failed. They were randomized to receive the following 7-day therapy: 1, moxifloxacin $400 \mathrm{mg}$ q.d., esomeprazole $20 \mathrm{mg}$ b.i.d., and amoxicillin $1 \mathrm{~g}$ b.i.d.; and 2, esomeprazole $40 \mathrm{mg}$ b.i.d., tripotassium dicitrate bismuthate $300 \mathrm{mg}$ q.i.d., metronidazole $500 \mathrm{mg}$ t.i.d., and tetracycline $500 \mathrm{mg}$ q.i.d. Eradication rates, drug compliance, and sideeffect rates of each group were evaluated.

Results: The eradication rates were 75.6 and $83.8 \%$ with moxifloxacin triple therapy, and 54.5 and $72.7 \%$ with quadruple therapy by intention-to-treat $(p=.042)$ and per-protocol analyses $(p=.260)$, respectively. Moxifloxacin triple therapy was significantly superior to quadruple therapy in terms of side-effect rates $(p=.039)$. Compliance for therapy, i.e., the percentage of tablets taken $(>85 \%)$, was 90.2 and $75.0 \%$, numerically higher in moxifloxacin triple therapy group than in quadruple therapy group, but without statistical difference $(p=.065)$.

Conclusions: Moxifloxacin-based triple therapy showed high eradication rates with few side effects and good drug compliance, suggesting this regimen could be a safe and effective option as second-line therapy for H. pylori infection in Korea.
\end{abstract}

Helicobacter pylori infection plays the major role in the pathogenesis of peptic ulcer disease, chronic gastritis, gastric mucosa-associated lymphoid tissue (MALT) lymphoma, and in gastric cancer [1]. Therefore, eradication of the bacteria has become the main therapy for these diseases. Worldwide consensus conferences have recommended triple therapy consisting of a proton pump inhibitor (PPI) and two antibiotics, mainly amoxicillin and clarithromycin, as first-line eradication therapy $[2,3]$. Although this treatment has been shown to be effective in numerous clinical trials, several meta-analysis revealed that the rates of eradication were widely variable, ranging from 70 to
95\% [3-5]. Second-line H. pylori eradication therapy is necessary for patients with first-line failure, for whom the Maastricht 2-2000 Consensus Report recommended the use of minimum 7-day, PPI, bismuth, metronidazole and tetracycline-based quadruple therapy [2]. However, this regimen may vary in efficacy in different patient populations. Although a recent meta-analysis showed that such treatment for 10-14 days is highly effective [6], in some countries or areas including Korea, the quadruple therapy has been also associated with eradication failure in more than $20 \%$ of cases [5,7-10]. The main causes of treatment failures in these areas compared with Western 
countries are thought to be poor patients' compliance and bacterial resistance [5,8-11]. The quadruple therapy entails complicated dosing schedules in terms of the number of tablets and interval of administrations. Moreover, considerable side-effect rates of metronidazole and tetracycline reduce patients' compliance [12]. Regarding bacterial resistance, $H$. pylori resistance to metronidazole has a negative impact on quadruple therapy $[13,14]$. High resistance rate of $H$. pylori to metronidazole worldwide, especially in Korea [15-20], has made the efficacy of metronidazole-containing quadruple therapy limited as a second-line therapy. Recent Korean reports revealed the eradication rates of a metronidazole- and tetracyclinecontaining second-line therapy were lower than $65 \%$ by intention-to-treat (ITT) analysis and $80 \%$ by per-protocol (PP) analysis [8-10]. Consequently, many therapeutic options with favorable compliance and various eradication rates are under trial [21-23], but ideal rescue regimens for a first $H$. pylori eradication failure are still uncertain.

The new generation fluoroquinolones have shown some favorable efficacies in the treatment of H. pylori [24-26], especially in terms of safety, simplicity of dosing schedule, and high efficacy as the initial eradication therapy. Recently, we have experienced excellent results concerning efficacy and tolerability using moxifloxacin-based triple therapy in small series of patients with two successive eradication failures [8]. With this background, we investigated whether this moxifloxacin-based triple therapy could be a strong candidate for the second-line treatment in terms of the efficacy and tolerability.

\section{Patients and Methods}

\section{Patients}

We consecutively enrolled patients, in whom a first eradication trial with PPI-amoxicillin-clarithromycin failed to eradicate H.pylori infection, at Seoul National University Bundang Hospital in Korea. Patients with concurrent critical illnesses, a history of previous upper gastrointestinal surgery, contraindication to any of the study medication, recent frequent intake of nonsteroidal anti-inflammatory drugs (NSAIDs), anticoagulants or steroids, an allergy to the study medications, and who were pregnant or breast-feeding women were excluded from the study. Other exclusion criteria included recent use of antimicrobials and any condition probably associated with poor compliance such as drug abusers or alcoholics. All patients enrolled in the study were aware of the study protocol and agreed to comply with the follow-up schedule, and all provided written informed consent. The study was approved by the Ethics Committee of Seoul National University Bundang Hospital.

\section{Methods}

Patients were considered as initial treatment failure if any of histology, rapid urease, or ${ }^{13} \mathrm{C}$-urea breath testing was positive before study. They were randomly assigned to one of the following two l-week treatment regimens: 1, MEA: moxifloxacin $400 \mathrm{mg}$ q.d., amoxicillin $1000 \mathrm{mg}$ b.i.d., and esomeprazole $20 \mathrm{mg}$ b.i.d.; and 2, EBMT: esomeprazole $20 \mathrm{mg}$ b.i.d., tripotassium dicitrate bismuthate $300 \mathrm{mg}$ q.i.d., metronidazole $500 \mathrm{mg}$ t.i.d., and tetracycline $500 \mathrm{mg}$ q.i.d. One week after the completion of therapy, compliance with therapy was evaluated by a physician both by being questioned and by pill count. The drug compliance was considered good if drug intake was more than $85 \%$. All patients were interviewed for adverse events at the same time. H. pylori eradication was defined as a negative ${ }^{13} \mathrm{C}$-urea breath test 4 weeks after the completion of therapy.

\section{${ }^{13} \mathrm{C}$-Urea Breath Test}

The patient fasted for 4 hours before the test. No test meal was given, and a predose breath sample was obtained. Seventy-five micrograms of ${ }^{13} \mathrm{C}$-urea powder (Helikit $^{\mathrm{TM}}$, Isotechnika, Edmonton, Canada) dissolved in $50 \mathrm{ml}$ of water was administered orally. The second breath sample was collected at 30 minutes. The cut-off value used was 4\%o [27]. Collected samples were analyzed by means of isotope ratio mass spectrometer (Heliview ${ }^{\circledR}$, Medichems, Seoul, Korea).

\section{Statistical Analysis}

Analyses of $H$. pylori eradication efficacy were performed on an ITT basis (included all patients enrolled in the study) and on a PP basis (excluding patients with poor compliance for therapy and unavailable data after therapy). The $95 \%$ confidence intervals (CI) for the differences of eradication rates between the two groups were calculated using standard methods. Continuous variables were analyzed by Student's $t$-test and categorical variables using the $\chi^{2}$ test or Fisher's exact test. Analyses were performed using SPSS (Statistical Package for the Social Sciences version 1 1.0; SPSS Inc., Chicago, IL, USA). $p<.05$ was considered to be statistically significant.

\section{Results}

\section{Patient Population}

Eighty-five patients were enrolled in the study from June 2004 through April 2005. Of these, 41 patients were randomized to the MEA group and 44 patients to the EBMT group. The baseline characteristics were similar in 


\begin{tabular}{llll}
\hline & MEA group & EBMT group & $p$ value \\
\hline Included in ITT analysis (n) & 41 & 44 & \\
Male/female (n) & $24 / 17$ & $23 / 21$ & $.562^{\mathrm{a}}$ \\
Mean age \pm SD (years) & $54.3 \pm 11.7$ & $51.6 \pm 12.5$ & $.295^{\mathrm{b}}$ \\
Disease $(\mathrm{n})$ & & & $.932^{\mathrm{a}}$ \\
$\quad$ Gastric ulcer & 11 & 11 & \\
Duodenal ulcer & 20 & 24 & \\
Gastroduodenal ulcer & 2 & 1 & $.316^{\mathrm{c}}$ \\
$\quad$ Gastric adenoma or cancer & 4 & 3 & \\
$\quad$ Nonulcer dyspepsia & 4 & 5 & \\
F/U loss or inadequate UBT assessment $(\mathrm{n})$ & 3 & 7 & \\
\hline
\end{tabular}

Table 1 Baseline demographic characteristics of patients

ITT, intention-to-treat; SD, standard deviation; F/U, follow-up; UBT, ${ }^{13} \mathrm{C}$-urea breath test;

MEA, moxifloxacin + esomeprazole + 'amoxicillin; EBMT, esomeprazole + bismuth + metronidazole

+ tetracycline.

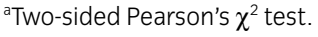

${ }^{\mathrm{b}}$ Two-tailed $t$-test.

'Two-sided Fisher's exact test.

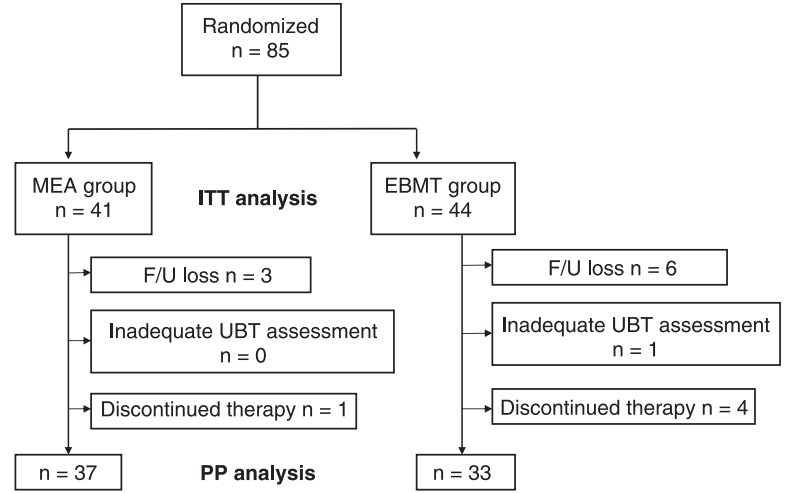

Figure 1 Flow diagram of the progress of patients included in intentionto-treat and per-protocol analysis. ITT, intention-to-treat; PP, per-protocol; MEA, moxifloxacin + esomeprazole + amoxicillin; EBMT, esomeprazole + bismuth + metronidazole + tetracycline; F/U, follow-up; UBT, ${ }^{13} \mathrm{C}$-urea breath test.

the two treatment groups (Table 1). All of them were included in the ITT analysis. Fifteen patients were excluded from the PP analysis; that is, follow-up loss and inadequate post-treatment assessment (no final UBT result) in 10 patients and protocol violation in five patients (the discontinuation of medication due to adverse events). Thus, 37 patients in the MEA group and 33 patients in the EBMT group comprised the study group for inclusion in the PP analysis. Figure 1 shows the disposition of all patients in these two groups.

\section{H. pylori Eradication Rates}

The eradication rates of $H$. pylori at the end of follow-up phase were $75.6 \%$ (95\% CI) in the MEA group and 54.5\%
Table 2 Helicobacter pylori eradication rates

\begin{tabular}{llll}
\hline & \multicolumn{1}{c}{ MEA } & EBMT & p value \\
\hline $\begin{array}{l}\text { ITT analysis } \\
\text { Eradication rate }\end{array}$ & $75.6 \%(31 / 41)$ & $54.5 \%(24 / 44)$ & $.042^{\text {a }}$ \\
$95 \% \mathrm{Cl}$ & $62.5-88.7 \%$ & $39.8-69.2 \%$ & \\
PP analysis & & & \\
$\quad$ Eradication rate & $83.8 \%(31 / 37)$ & $72.7 \%(24 / 33)$ & $.260^{\text {a }}$ \\
$95 \% \mathrm{Cl}$ & $71.9-95.7 \%$ & $55.7-89.7 \%$ & \\
\hline
\end{tabular}

ITT, intention-to-treat; PP, per-protocol; Cl, confidence interval; MEA, moxifloxacin + esomeprazole + amoxicillin; EBMT, esomeprazole + bismuth + metronidazole + tetracycline.

aTwo-sided Pearson's $\chi^{2}$ test.

in the EBMT group by ITT analysis (Table 2). Based on the PP analysis, $H$. pylori eradication was achieved in $83.8 \%$ in the MEA group and $72.7 \%$ in the EBMT group. H. pylori eradication rates in the MEA group were significantly higher than in the EBMT group by ITT analysis $(p=.042)$, but not by PP analysis $(p=.260)$.

\section{Adverse Events and Compliance}

Adverse events were described in 4 of 41 patients $(9.8 \%)$ in the MEA group and in 12 of 44 patients $(27.3 \%)$ in the EBMT group; the most common one was epigastric discomfort (Table 3). Side-effect rate in the MEA group was significantly lower than in the EBMT group $(p=.039)$. Most adverse events were mild to moderate in intensity. Five serious adverse events were reported and they were dropped out due to the adverse events. Of these, one patient was in the MEA group $(2.4 \%)$ and the adverse symptom was epigastric pain, and four patients were in 
Table 3 Adverse events

\begin{tabular}{llc}
\hline Adverse events & $\begin{array}{c}\text { MEA group } \\
(\mathrm{n}=41)\end{array}$ & $\begin{array}{c}\text { EBMT group } \\
(\mathrm{n}=44)\end{array}$ \\
\hline $\begin{array}{l}\text { Epigastric discomfort } \\
\text { Nausea or vomiting }\end{array}$ & $2(4.9 \%)$ & $6(13.6 \%)$ \\
Facial spasm or paresthesia & $1(2.4 \%)$ & $2(4.5 \%)$ \\
Diarrhea & - & $2(4.5 \%)$ \\
Chest tightness & $1(2.4 \%)$ & - \\
Constipation & - & $1(2.3 \%)$ \\
Total & - & $1(2.3 \%)$ \\
\hline
\end{tabular}

MEA, moxifloxacin + esomeprazole + amoxicillin; EBMT, esomeprazole + bismuth + metronidazole + tetracycline.

${ }^{a} p<.05$ when compared with the EBMT group (two-sided Pearson's $\chi^{2}$ test).

the EBMT group (9.1\%); two patients had epigastric pain, and the other two showed vomiting and paresthesia, respectively.

Compliance for therapy, i.e., the percentage of tablets taken $(>85 \%)$, was $90.2 \%$ (37/41 patients) and $75.0 \%$ (33/44 patients) in the MEA and EBMT groups, which was not significantly different between the two groups $(p=.065)$.

\section{Discussion}

Quadruple therapy containing PPI, bismuth, tetracycline, and metronidazole has been generally used as a secondline therapy after initial eradication failure and chosen as the recommended regimen in several guidelines $[2,3]$. However, this regimen has been associated with eradication failure in more than $20 \%$ of the cases by PP analysis in some areas including Korea, the main cause of which is thought to be bacterial resistance. Moreover, poor patients' compliance due to considerable side-effect rates and complicated dosing schedules has decreased the ITT eradication rates. To overcome the bacterial resistance and poor compliance, many potential alternative therapeutic options with favorable compliance and various eradication rates have been undertaken [21-23,28-30], but the selection of an appropriate second-line therapy is still challenging for the clinician.

The background of our study was that moxifloxacin, which has several advantages in terms of safety and simplicity of dosing schedules, could decrease the dropout of patients during eradication therapy resulting in increase of the eradication by ITT analysis. In addition, in vitro studies show excellent susceptibility of $H$. pylori to newer quinolones including moxifloxacin [24,31,32]. In vitro susceptibility study performed in our hospital also revealed low resistance rate of $H$. pylori against moxifloxacin, 5.6\%
$(3 / 54)$ in comparison with $40.7 \%(22 / 54)$ of metronidazole (unpublished data), supporting these data. Thus, we hypothesized that the PP eradication rate of moxifloxacincontaining regimen could be higher or at least equal to that of conventional quadruple therapy as a second-line therapy. To prove this, we compared the efficacy and tolerability of moxifloxacin-based triple therapy with those of conventional quadruple therapy for the treatment of H.pylori infection after failure of a first-line eradication. The ITT and PP H.pylori eradication rates turned out to be $75.6 /$ $83.8 \%$ for the moxifloxacin-based triple therapy and 54.5/ $72.7 \%$ for the quadruple therapy. The eradication rate of triple therapy was significantly higher than that of the quadruple therapy by ITT analysis. Drug side effects were less common in the triple therapy than in the quadruple therapy, which might have influenced on the numerical difference in drug compliance and follow-up loss between the two groups. This study suggests that moxifloxacinbased triple regimen is efficacious and tolerable and could be, therefore, used as an alternative second-line eradication regimen instead of standard quadruple therapy for patients who have failed in the initial eradication therapy.

To overcome resistance problems, at least, the evaluation of drugs for a second-line H. pylori therapy without cross-resistance to nitroimidazoles or macrolides as components of retreatment combination therapies seems to be worthwhile. In addition to moxifloxacin, several antibiotics are suggested as candidates satisfying these conditions. Rifabutin, another potential candidate antibiotic, has been proven to exhibit high in vitro activity against $H$. pylori $[33,34]$, and rifabutin-based clinical trials have suggested that rifabutin may be a promising second-line or rescue antibiotics for $H$. pylori eradication [22,35]. However, several concerns remain about its use as a second-line $H$. pylori therapy especially in Korea, which is still a high endemic area of tuberculosis. Its high cost, rare but serious side effect, i.e., neutropenia and thrombocytopenia, and most of all, some concern about the emergence of multidrugresistant Mycobacterium tuberculosis limits its use to patients who have experienced multiple failures of $H$. pylori eradication therapies $[21,35]$. Besides changing the antibiotics, it is evident that the prolongation of treatment duration could cope with resistance problems. A recent meta-analysis suggested 14-day, metronidazole-containing quadruple therapy could be recommended in first-line as well as second-line anti-H. pylori treatment [6]. It might be valuable to compare this moxifloxacin-based triple therapy with 14-day quadruple therapy in the future if the sideeffects and complexity of 14-day quadruple therapy could be overcome in Korea.

Our study has a limitation in that we did not evaluate the antibiotic resistance in relation to treatment regimens and eradication rates. The utility of the culture with 
subsequent antibiotic susceptibility testing and the moment when it should be performed after eradication failure are still controversial. However, it gains more appraisal that culture after a first eradication failure is not necessary $[3,7,21]$. It may be pertinent to perform the bacterial culture and antibiotic susceptibility testing after two or more successive failures of eradication. From a practical viewpoint, we omitted the culture study. Another potential weakness of our study is that the compliance of quadruple therapy group was poor. The main reason of poor compliance of quadruple therapy is likely to have originated from the complexity of tablet-taking times (two, three, four times per drug) and the high tablet number of this regimen in Korea. That is, quadruple therapy consists of esomeprazole $20 \mathrm{mg}$ b.i.d. (2 tablets), tripotassium dicitrate bismuthate $300 \mathrm{mg}$ q.i.d. (4 tablets), metronidazole $500 \mathrm{mg}$ t.i.d. (250 mg, 6 tablets), and tetracycline $500 \mathrm{mg}$ q.i.d. (250 mg, 8 tablets) in comparison of MEA: moxifloxacin $400 \mathrm{mg}$ q.d. (1 tablet), amoxicillin $1000 \mathrm{mg}$ b.i.d. (250 mg, 4 tablets), and esomeprazole $20 \mathrm{mg}$ b.i.d. (2 tablets). The tablet number of quadruple therapy is 20, nearly three times more than that of MEA, which has seven. In addition, we met the patients only two times during the second treatment, at the start point of study and after the completion of regimen, which is reflecting the usual practice pattern. This was equally applied to both treatment groups, and the compliance was not statistically different between moxifloxacin-based therapy and quadruple therapy.

In conclusion, l-week esomeprazole, amoxicillin, moxifloxacin-based triple therapy was highly effective for a second-line H. pylori eradication therapy, especially, in terms of safety and tolerability. Further larger studies are needed to determine the broad application of this eradication regimen in comparison with currently approved quadruple, second-line therapy.

This work was supported by grant 02-03-001 from SNUBH research fund.

\section{References}

1 Suerbaum S, Michetti P. Helicobacter pylori infection. N Engl J Med 2002;347:1175-86.

2 Malfertheiner P, Megraud F, O'Morain C, Hungin AP, Jones R, Axon A, Graham DY, Tytgat G; European Helicobacter Pylori Study Group (EHPSG). Current concepts in the management of Helicobacter pylori infection - the Maastricht 2-2000 Consensus Report. Aliment Pharmacol Ther 2002;16:167-80.

3 Lam SK, Talley NJ. Report of the 1997 Asia Pacific Consensus Conference on the management of Helicobacter pylori infection. $J$ Gastroenterol Hepatol 1998;13:1-12.

4 Huang JQ, Hunt RH. Treatment after failure: the problem of 'non-responders'. Gut. 1999;45(Suppl. 1):I40-4.

5 Laheij RJ, Rossum LG, Jansen JB, Straatman H, Verbeek AL. Evaluation of treatment regimens to cure Helicobacter pylori infection - a meta-analysis. Aliment Pharmacol Ther 1999;13:857-64.

6 Fischbach LA, van Zanten S, Dickason J. Meta-analysis: the efficacy, adverse events, and adherence related to first-line anti-Helicobacter pylori quadruple therapies. Aliment Pharmacol Ther 2004;20:1071-82.

7 Gisbert JP, Pajares JM. Helicobacter pylori therapy: first-line options and rescue regimen. Dig Dis 2001;19:134-43.

8 Cheon JH, Kim N, Lee DH, et al. Trial of moxifloxacin-containing triple therapy after initial and second-line treatment failures for Helicobacter pylori infection. Korean J Gastroenterol 2005;45:111-7.

9 Chung WC, Cho YS, Jeong JJ, Lee IS, Kim SW, Yang JM, Choi MG, Chung IS, Park DH. Eradication rate of Helicobacter pylori infection according to the diseases, therapeutic regimens, and reinfection rate after successful eradication in a tertiary clinic. Korean $J$ Gastroenterol 2003;41:1-8.

10 Park MJ, Choi IJ, Kim JS, Lee DH, Jung HC, Song IS, Kim CY. Efficacy of quadruple therapy as retreatment regimen in Helicobacter pylori-positive peptic ulcer disease. Korean $J$ Gastroenterol 2000;36:457-64.

11 Mantzaris GJ, Petraki K, Archavlis E, Amberiadis P, Christoforidis P, Kourtessas D, Chiotakakou E, Triantafyllou G. Omeprazole triple therapy versus omeprazole quadruple therapy for healing duodenal ulcer and eradication of Helicobacter pylori infection: a 24-month follow-up study. Eur J Gastroenterol Hepatol 2002;14:1237-43.

12 Ko JJ, Kim N, Oh JH, Lee CG, Ko YH, Lim SH, Lee KH. Triple therapy for eradication of Helicobacter pylori in patients with peptic ulcer. Korean J Gastroenterol 1998;31:605-14.

13 Chi CH, Lin CY, Sheu BS, Yang HB, Huang AH, Wu JJ. Quadruple therapy containing amoxicillin and tetracycline is an effective regimen to rescue failed triple therapy by overcoming the antimicrobial resistance of Helicobacter pylori. Aliment Pharmacol Ther 2003;18:347-53.

14 Lamouliatte H, Megraud F, Delchier JC, et al. Second-line treatment for failure to eradicate Helicobacter pylori: a randomized trial comparing four treatment strategies. Aliment Pharmacol Ther 2003;18:791-7.

15 Kim JJ, Reddy R, Lee M, Kim JG, El-Zaatari FA, Osato MS, Graham DY, Kwon DH. Analysis of metronidazole, clarithromycin and tetracycline resistance of Helicobacter pylori isolates from Korea. J Antimicrob Chemother 2001;47:459-61.

16 Kim JM, Kim JS, Jung HC, Kim N, Kim YJ, Song IS. Distribution of antibiotic MICs for Helicobacter pylori strains over a 16-year period in patients from Seoul, South Korea. Antimicrob Agents Chemother 2004;48:4843-7.

17 Eun CS, Han DS, Park JY, Jeon YC, Hahm JS, Kim KS, Kang JO. Changing pattern of antimicrobial resistance of Helicobacter pylori in Korean patients with peptic ulcer diseases. J Gastroenterol 2003;38:436-41.

18 Nahar S, Mukhopadhyay AK, Khan R, et al. Antimicrobial susceptibility of Helicobacter pylori strains isolated in Bangladesh. J Clin Microbiol 2004;42:4856-8.

19 Sherif M, Mohran Z, Fathy H, Rockabrand DM, Rozmajzl PJ, Frenck RW. Universal high-level primary metronidazole resistance in Helicobacter pylori isolated from children in Egypt. J Clin Microbiol 2004;42:4832-4.

20 Samra Z, Shmuely H, Niv Y, et al. Resistance of Helicobacter pylori isolated in Israel to metronidazole, clarithromycin, tetracycline, amoxicillin and cefixime. J Antimicrob Chemother. 2002;49:1023-6.

21 Gisbert JP, Pajares JM. Helicobacter pylori 'rescue' regimen when proton pump inhibitor-based triple therapies fail. Aliment Pharmacol Ther 2002;16:1047-57. 
22 Wong WM, Gu Q, Lam SK, et al. Randomized controlled study of rabeprazole, levofloxacin and rifabutin triple therapy vs. quadruple therapy as second-line treatment for Helicobacter pylori infection. Aliment Pharmacol Ther 2003; 17:553-60.

23 Miehlke S, Kirsch C, Schneider-Brachert W, et al. A prospective, randomized study of quadruple therapy and high-dose dual therapy for treatment of Helicobacter pylori resistant to both metronidazole and clarithromycin. Helicobacter 2003;8:310-9.

24 Di Caro S, Ojetti V, Zocco MA, et al. Mono, dual and triple moxifloxacin-based therapies for Helicobacter pylori eradication. Aliment Pharmacol Ther 2002;16:527-32.

25 Cammarota G, Cianci R, Cannizzaro O, et al. Efficacy of two one-week rabeprazole/levofloxacin-based triple therapies for Helicobacter pylori infection. Aliment Pharmacol Ther 2000;14:1339_ 43.

26 Sharara AI, Chaar HF, Racoubian E, Moukhachen O, Barada KA, Mourad FH, Araj GF. Efficacy of two rabeprazole/gatifloxacinbased triple therapies for Helicobacter pylori infection. Helicobacter 2004;9:255-61

27 Mock T, Yatscoff R, Foster R, Hyun JH, Chung IS, Shim CS, Yacyshyn B. Clinical validation of the Helikit: $a{ }^{13} \mathrm{C}$ urea breath test used for the diagnosis of Helicobacter pylori infection. Clin Biochem 1999;32:59-63.

28 Perri F, Festa V, Merla A, Barberani F, Pilotto A, Andriulli A. Randomized study of different 'second-line' therapies for
Helicobacter pylori infection after failure of the standard 'Maastricht triple therapy'. Aliment Pharmacol Ther 2003;18:815-20.

29 Katelaris PH, Forbes GM, Talley NJ, Crotty B. A randomized comparison of quadruple and triple therapies for Helicobacter pylori eradication: The QUADRATE Study. Gastroenterology 2002;123:1763-9.

30 Bilardi C, Dulbecco P, Zentilin P, et al. A 10-day levofloxacinbased therapy in patients with resistant Helicobacter pylori infection: a controlled trial. Clin Gastroenterol Hepatol. 2004;2:997-1002.

31 Bauernfeind A. Comparison of the antibacterial activities of the quinolones Bay 12-8039, gatifloxacin (AM 1155), trovafloxacin, clinafloxacin, levofloxacin and ciprofloxacin. J Antimicrob Chemother 1997;40:639-51.

32 Iwao E, Yokoyama Y, Yamamoto K, Hirayama F, Haga K. In vitro and in vivo anti-Helicobacter pylori activity of Y-904, a new fluoroquinolone. J Infect Chemother 2003;9:165-71.

33 Akada JK, Shirai M, Fujii K, Okita K, Nakazawa T. In vitro anti-Helicobacter pylori activities of new rifamycin derivatives, KRM-1648 and KRM-1657. Antimicrob Agents Chemother 1999;43:1072-6.

34 Heep M, Beck D, Bayerdorffer E, Lehn N. Rifampin and rifabutin resistance mechanism in Helicobacter pylori. Antimicrob Agents Chemother 1999;43:1497-9.

35 Perri F, Festa V, Clemente R, Quitadamo M, Andriulli A. Rifabutin-based 'rescue therapy' for Helicobacter pylori infected patients after failure of standard regimens. Aliment Pharmacol Ther 2000;14:311-6. 